\title{
Influencia del control del estrés en el rendimiento deportivo: la autoconfianza, la ansiedad y la concentración en deportistas Influence of stress control in the sports performance: self-confidence, anxiety and concentration in athletes
}

*Gloria González Campos, **Pedro Valdivia-Moral, ***Javier Cachón Zagalaz, **Felix Zurita Ortega, ****Oscar Romero-Ramos

*Universidad de Sevilla (España), **Universidad de Granada (España), ***Universidad de Jaén (España), ****Universidad de Malága (España)

Resumen. En esta investigación se realiza un estudio sobre el control del estrés en deportistas, utilizando como muestra 100 jugadores de fútbol semiprofesionales. Se parte de la consideración de que controlar el estrés para no llegar a estados de ansiedad es imprescindible, ya que alcanzar estos estados provoca efectos negativos en otras variables psicológicas del deportista, disminuyendo su autoconfianza y perjudicando el control atencional. El objetivo de esta investigación es describir los niveles de ansiedad, autoconfianza y atención-concentración en futbolistas semiprofesionales, mediante el análisis de la variable psicológica control del estrés. El instrumento utilizado para el estudio es la escala control del estrés del cuestionario de Características Psicológicas relacionadas con el Rendimiento Deportivo (CPRD). Como conclusiones del estudio, cabe destacar que desarrollar la habilidad psicológica de control del estrés, previene alcanzar estados de ansiedad y como consecuencia, posibilita mantener elevada la autoconfianza y una buena capacidad de atención-concentración durante la competición.

Palabras Clave: habilidades psicológicas, estrés, ansiedad, autoconfianza, atención-concentración, fútbol.

Abstract. In this research, a study on stress management in athletes is carried out with a sample of 100 semi-professional football players. Stress control is essential for avoiding a state of anxiety, as this state may have negative effects on other psychological variables of athletes, decreasing their self-confidence and harming their attentional control. The objective of this research is to describe anxiety, self-confidence, and attention-concentration levels in semi-professional footballers by means of analysing stress control. The instrument used for the study is the stress control scale from the Psychological Characteristics related to Sports Performance (CPRD) questionnaire. As a conclusion, it is noteworthy that developing the psychological ability of controlling stress prevents the appearance of anxiety states, allowing athletes to maintain high self-confidence and good attentionconcentration capacity during competitions.

Key words: psychological skills, stress, anxiety, self-confidence, attention-concentration, football.

\section{Introducción}

Expertos en Psicología del deporte coinciden en que la combinación de elementos técnico-tácticos, físicos, fisiológicos y psicológicos en el deporte de rendimiento permiten competir con intensidad, a un alto ritmo de juego, afrontar presiones, etc. (Cox, 2009; Moore, Bullough, Goldsmith, \& Edmondson, 2014; Weinberg \& Gould, 2010), de manera que reforzar estos elementos supone una optimización del rendimiento en el deportista, en particular, si es capaz de canalizar positivamente el estrés y la ansiedad (García-Naviera \& Ruiz-Barquín, 2015).

Dentro de las variables psicológicas influyentes en la productividad deportiva para la consecución del éxito, Gimeno, Buceta \& PérezLlantada (2007), destacan la motivación, la atención, el estrés, la ansiedad, la autoconfianza, los estados de ánimo, el autocontrol y la autorregulación, la cohesión, las habilidades interpersonales o el ajuste emocional. Pazo, Sáenz-López \& Fradua (2012) recalcan que las habilidades psicológicas en el jugador son la base de su carrera deportiva, por lo que se hace imprescindible profundizar y obtener referencias sobre ello mediante técnicas e instrumentos psicológicos. Todo ello es transferible también al fútbol sala, según se desprende del estudio de CachónZagalaz, Rodrigo-Conde, Campoy-Aranda, Linares-Girela \& ZagalazSánchez (2012).

González, Garcés de los Fayos \& Ortega (2014) señalan que una elevada ansiedad se relaciona negativamente con el rendimiento deportivo, el bienestar y la salud del deportista. He aquí la importancia del desarrollo de habilidades psicológicas en el atleta con la finalidad de que sea capaz de canalizar positivamente el afrontamiento competitivo y éste sea fuente de disfrute.

La existencia de factores estresantes afectan negativamente al individuo a nivel personal y social, abordando su vulnerabilidad física, intelectual y psicosocial (López-Suárez, 2014; Romero, 2015). En el ámbito deportivo, el estrés es definido por Kent (2003:298) como «una afección psicológica que se produce cuando las personas observan un

Fecha recepción: 12-06-15. Fecha de aceptación: 27-10-16 Pedro Valdivia Moral

pvaldivia@ugr.es desequilibrio sustancial entre las exigencias que soportan y su capacidad para cumplirlas, y cuando dicha incapacidad tiene consecuencias importantes». Aunque Ferreira, Valdés \& González (2002) explican que el estrés también puede ser positivo y que prepara al cuerpo para mantener mayor capacidad de atención, motivación e incluso entusiasmo para afrontar demandas deportivas.

Sin embargo, la ansiedad es un estado emocional de tensión nerviosa y de miedo intenso, caracterizada por síntomas somáticos como temblor, inquietud, sudoración, hiperventilación, palpitaciones, etc., cuyos síntomas cognitivos son inquietud psíquica, hipervigilancia, pérdida de concentración y distorsiones cognitivas (Saz, 2000). En el deporte, Kent (2003) expone que un alto nivel de ansiedad reduce el rendimiento porque afecta a la calidad de la atención y como consecuencia, a la ejecución, destacando tres tipos de ansiedad: cognitiva, somática y conductual. En esta línea, González-Ramírez, Quezada-Berumen, Díaz-Rodríguez \& Cano-Vindel (2014) confirman que la ansiedad no puede tratarse como un constructo unitario sino multidimensional en el que interactúan variables cognitivas, fisiológicas y motoras. Así pues para su medición, Navlet (2012) llega a la conclusión de que para que sea específica se ha de estudiar la ansiedad como proceso emocional, actitud emocional cognitiva, rasgo de personalidad y trastorno.

Por su parte, la autoconfianza es la convicción de que se puede ejecutar con éxito la conducta requerida y producir un resultado, por lo que en el ámbito deportivo se utiliza para referirse a la autopercepción de la capacidad para enfrentarse a una tarea (Nicolás, 2009). Según Telletxea (2007), la autoconfianza es puesta a prueba reiteradamente en campos altamente competitivos, de manera que después de fracasos o contratiempos en el deporte, pueden aparecer dudas sobre uno mismo.

González-Campos, Valdivia-Moral, Zagalaz \& Romero (2014) destacan la importancia de la capacidad de resiliencia en el deporte, que según Ruiz, De la Vega, Poveda, Rosado \& Serpa (2012), este concepto tiene una gran difusión investigadora en el ámbito deportivo (Gucciardi, Gordon \& Dimmarck, 2009; Hosseini \& Besharat, 2010; De la Vega, Rivera \& Ruiz, 2011). Un deportista con poca resiliencia es psicológicamente vulnerable. Hay deportistas que absorben ansiedades, inquietudes, presiones, mientras otros son refractarios a estas contingencias (Dramisino, 2007). No obstante, un estudio realizado por Chacón, 
Castro-Sánchez, Espejo-Garcés \& Zurita (2016) demuestra que los futbolistas son los que menos puntúan en la capacidad de resiliencia.

Otra variable psicológica a destacar es la atención-concentración. La atención es según Dosil (2004), la forma de interacción con el entorno, en la que el sujeto establece contacto con los estímulos relevantes de la situación, procurando desechar los estímulos no relevantes. En cuanto a la concentración, Dosil (2004:178) la define como «el mantenimiento de las condiciones atencionales a lo largo de un tiempo más o menos duradero». En el fútbol, el jugador debe ser capaz tanto de seleccionar el foco atencional, como de oscilar de un foco a otro frecuentemente (García, Rodríguez \& Garzón, 2011).

En la competición existe un gran número de estresores que se presentan con frecuencia, por lo que González (2011) advierte de la importancia de saber controlar el estrés, para que la inmediatez que se requiere para la obtención de buenos resultados no irrumpa en el equilibrio mental y emocional del deportista. La influencia de la ansiedad en el rendimiento deportivo es patente, llevando a los investigadores a profundizar en su estudio. Lundqvist, Kenttä \& Raglin (2011) afirman que puntuaciones bajas en esta variable y combinados con altos niveles de autoconfianza son los que funcionan como facilitadores en el logro deportivo. En definitiva, canalizar positivamente el estrés a lo largo de la carrera deportiva y crearse un ímpetu psicológico positivo influirá para desarrollar un rendimiento óptimo deportivo (González-Campos, Valdivia-Moral, Zagalaz \& Romero, 2015).

Otra variable relacionada con el estrés y con la autoconfianza es la resiliencia. En el fútbol, Ruiz et al. (2012) analizan esta variable en 110 futbolistas y la comparan con un estudio de Vigário, Serpa \& Rosado (2009) que previamente examinaron esta capacidad en una muestra de estudiantes. Para ello, aplican el cuestionario adaptado al castellano «Escala de Resiliencia» pertenecientes a un club de fútbol profesional de la provincia de Córdoba y describen valores mayores de resiliencia global en futbolistas que en los estudiantes.

En esta investigación se realiza un estudio sobre el control del estrés en deportistas, utilizando como muestra jugadores de fútbol semiprofesionales. Se parte de la consideración de que controlar el estrés para no llegar a estados de ansiedad es imprescindible, ya que alcanzar estados de ansiedad hace que descienda la autoconfianza y el control atencional. Estudios confirmatorios de esta consideración lo encontramos en Ruiz-Juan \& Zarauz (2013) ratificando que los estados de ansiedad correlacionan significativa y negativamente con la autoconfianza (Buceta, López, Pérez-Llantada, Vallejo \& Del Pino, 2003; Ruiz-Juan \& Zarauz, 2014).

El objetivo de esta investigación es describir los niveles de ansiedad, autoconfianza y atención-concentración en futbolistas semiprofesionales, mediante el análisis de la variable psicológica control del estrés.

\section{Material y Método}

\section{Participantes}

La muestra está compuesta por 100 jugadores de fútbol semiprofesionales que compiten en la categoría de tercera división del fútbol español en la temporada 2015/2016. Debido a que en las categorías senior del fútbol español se compite por sexos, toda la población son hombres. La muestra $(\mathrm{n}=100)$ ha sido seleccionada mediante un muestreo aleatorio entre equipos de tercera división pertenecientes a las provincias de Sevilla y Jaén. Está compuesta por un jugador uruguayo, un marroquí, tres rumanos y el resto son españoles. La edad de los futbolistas oscila entre 17 y 24 años con una media de 20.9 .

\section{Instrumento}

Para este estudio se ha utilizado la escala control del estrés del cuestionario de Características Psicológicas relacionadas con el Rendimiento Deportivo (CPRD) de Buceta, Gimeno \& Pérez-Llantada (1994). Está compuesto por 55 ítems distribuidos en cinco escalas: control del estrés, influencia de la evaluación del rendimiento, motivación, habilidad mental y cohesión de equipo.

Los ítems están formulados en una escala tipo Likert desde «total- mente en desacuerdo» a «totalmente de acuerdo» de cero a cuatro puntos.

El instrumento, según sus autores tiene una fiabilidad de .85 en su conjunto, y en la escala de control del estrés ofrece una consistencia interna de .88. En nuestro estudio el CPRD en su totalidad ha mostrado un nivel deAlpha de Cronbach de .726, ajustándose a las recomendaciones de Nunnally (1970). Para ello, fue necesario excluir el ítem 24 (correspondiente a la subescala autoconfianza) y el ítem 30 (perteneciente a la subescala ansiedad).

La escala control del estrés recoge respuestas del deportista, por una parte, relacionadas con estados de tensión, concentración y confianza en cuanto a las demandas del entrenamiento y la competición, y por otra, respuestas sobre situaciones cargadas de estrés antes, durante y después de competir.

La escala original se compone de 20 ítems que se corresponden con tres subescalas: control de la ansiedad, autoconfianza y atención-concentración. De los 20 ítems, cinco hacen referencia al constructo ansiedad. Diez están relacionados con la autoconfianza, y cinco con la atención-concentración. Es necesario volver a indicar que fueron eliminados dos ítems para elevar el Alpha de Cronbach.

Una puntuación alta en esta escala indica que el deportista utiliza sus habilidades psicológicas positivamente, es decir, que confía en sus posibilidades, no manifiesta ansiedad para competir y es capaz de mantener su atención focalizada en aspectos relevantes del juego mientras compite.

\section{Diseñoy Procedimiento}

Este estudio tiene un carácter descriptivo y un enfoque cuantitativo como se puede comprobar en los posteriores resultados. Así mismo, hay que destacar que se trata de un trabajo transversal ya que recoge datos en un momento puntual de la temporada.

Durante las primeras semanas del inicio de la Liga española de la temporada 2015-16, el cuestionario fue cumplimentado por los jugadores de los diferentes equipos seleccionados de manera aleatoria para este estudio, bajo la información de una especialista en Psicología del deporte y sus ayudantes, explicando las pautas a seguir y solicitando objetividad y sinceridad en las respuestas.

Todos los participantes fueron informados tanto del objetivo del estudio y de la absoluta confidencialidad de las respuestas otorgadas, como del manejo posterior de los datos, con la finalidad de su mejora individual y colectiva. Todos los participantes dieron su consentimiento a participar en el estudio una vez que fueron informados. En el caso de aquellos que eran menores de edad, se aportó el consentimiento de los padres.

\section{Análisis de Datos}

Los datos fueron analizados con el programa estadístico SPSS versión 22.0. Se comprobó la fiabilidad interna del instrumento medianteel cálculo del Alpha de Cronbach. Acontinuación se realizaron análisis de frecuencias, cálculos de medias y desviaciones típicas para la estadística descriptiva y la prueba ANOVA de un factor para establecer las diferencias significativas entre las distintas variables.

\begin{tabular}{|c|c|c|c|c|c|}
\hline $\begin{array}{c}\text { Control del Estrés } \\
\text { Ansiedad }\end{array}$ & $\begin{array}{c}\text { Totalmente en } \\
\text { desacuerdo }\end{array}$ & $\begin{array}{c}\text { En } \\
\text { desacuerdo } \\
\end{array}$ & Indiferente & De acuerdo & $\begin{array}{l}\text { Totalmente } \\
\text { de Acuerdo }\end{array}$ \\
\hline \multicolumn{6}{|l|}{ 6. Rara vez me encuentro tan } \\
\hline tenso como para que mi & 13 & 30 & 25 & 27 & 5 \\
\hline $\begin{array}{l}\text { negativamente en mi } \\
\text { rendimiento }\end{array}$ & $13 \%$ & $30 \%$ & $25 \%$ & $27 \%$ & $5 \%$ \\
\hline \multicolumn{6}{|l|}{ 12. A menudo estoy "muerto } \\
\hline de miedo" en los momentos & 73 & 14 & 5 & 4 & 4 \\
\hline $\begin{array}{l}\text { anteriores al comenzo de mı } \\
\text { participación en una } \\
\text { competición }\end{array}$ & $73 \%$ & $14 \%$ & $5 \%$ & $4 \%$ & $4 \%$ \\
\hline $\begin{array}{l}\text { 20. Gasto mucha energía } \\
\text { intentando estar tranquilo }\end{array}$ & & & 11 & 4 & 4 \\
\hline $\begin{array}{l}\text { Intentando estar tranquilo } \\
\text { antes de que comience una } \\
\text { competición }\end{array}$ & $\begin{array}{l}36 \\
37.5 \%\end{array}$ & $42.7 \%$ & $11.5 \%$ & $\begin{array}{c}4 \\
4.2 \%\end{array}$ & $\begin{array}{c}4 \\
4.2 \%\end{array}$ \\
\hline $\begin{array}{l}\text { 36. El día anterior a una } \\
\text { competición me encuentro }\end{array}$ & 35 & 36 & 8 & 17 & 44 \\
\hline $\begin{array}{l}\text { habitualmente demasiado } \\
\text { nervioso o preocupado }\end{array}$ & $35 \%$ & $36 \%$ & $8 \%$ & $17 \%$ & $4 \%$ \\
\hline
\end{tabular}




\section{Resultados}

Tras los análisis estadísticos pertinentes, a continuación se exponen los resultados en función de la escala analizada. En la tabla 1 se muestran los datos obtenidos según la dimensión control del estrés y su subescala ansiedad. Como se puede comprobar la mayoría de jugadores están totalmente en desacuerdo cuando se les consulta si tienen miedo a la hora de comenzar una competición. Por otro lado, la mayoría de los encuestados se muestran en desacuerdo o totalmente en desacuerdo cuando se les consulta si el día anterior a una competición están nerviosos o preocupados.

A continuación, en la tabla 2 se muestran los resultados de la dimensión control del estrés y su subescala autoconfianza. Respecto a esta variable psicológica, los futbolistas encuestados resaltan los resultados mostrados en el ítem 3 , en el que se puede ver que los valores son medios cuando se les consulta si tienen confianza en su técnica. Solo un 18\% está totalmente de acuerdo, si bien nadie está totalmente en desacuerdo. Por otro lado, la gran mayoría de los encuestados están de acuerdo o totalmente de acuerdo cuando se les consulta si tienen fe en ellos mismos.

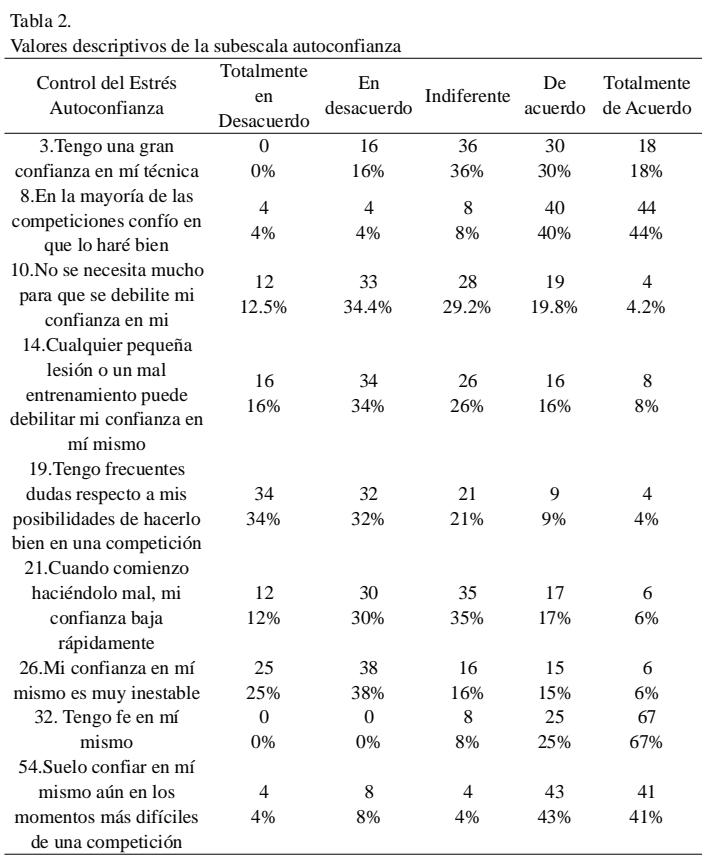

Por último, en la tabla 3 se recogen los resultados de la subescala atención-concentración. En la misma se puede apreciar que los deportistas encuestados manifiestan estar totalmente en desacuerdo o en desacuerdo sobre si suelen tener problemas concentrándose mientras compiten. Además, el 95\% está de acuerdo o totalmente de acuerdo en el ítem 43 que mide si se concentran con facilidad en lo más importante en cada momento de la competición, sin embargo, casi un $60 \%$ muestra que ante situaciones erráticas, sus niveles de atención-concentración descienden

En la siguiente tabla se muestran los valores de media y desviación estándar de las diferentes dimensiones.

Por último, tras realizar la prueba ANOVA se hallaron diferencias significativas entre las siguientes variables. Entre lavariable Autoconfianza y Atención-Concentración se encontró un valor de p=.014. En el caso deAtención-Concentración y Ansiedad se halló un valor de $\mathrm{p}=$.017. Por último entre la ansiedad y la autoconfianza no se hallaron diferencias significativas.

\section{Discusión}

Los resultados expuestos anteriormente responden al objetivo de la investigación y ponen de manifiesto la importancia de estos parámetros

\begin{tabular}{|c|c|c|c|c|c|}
\hline $\begin{array}{c}\text { Control del Estrés } \\
\text { Atención-Concentración }\end{array}$ & $\begin{array}{c}\text { Totalmente en } \\
\text { Desacuerdo }\end{array}$ & $\begin{array}{c}\text { En } \\
\text { desacuerdo }\end{array}$ & Indiferente & $\begin{array}{c}\text { De } \\
\text { acuerdo }\end{array}$ & $\begin{array}{l}\text { Totalmente } \\
\text { de Acuerdo }\end{array}$ \\
\hline $\begin{array}{c}\text { 1.Suelo tener problemas } \\
\text { concentrándome mientras } \\
\text { compito }\end{array}$ & $\begin{array}{c}29 \\
29 \%\end{array}$ & $\begin{array}{c}64 \\
64 \%\end{array}$ & $\begin{array}{c}7 \\
7 \%\end{array}$ & $\begin{array}{c}0 \\
0 \%\end{array}$ & $\begin{array}{c}0 \\
0 \%\end{array}$ \\
\hline $\begin{array}{l}\text { 13. Cuando cometo un error } \\
\text { me cuesta olvidarlo para }\end{array}$ & 8 & 33 & 25 & 19 & 15 \\
\hline $\begin{array}{l}\text { concentrarme rápidamente } \\
\text { en lo que tengo que hacer } \\
\text { 17.Durante mi actuación en } \\
\text { una competición mi }\end{array}$ & $8 \%$ & $33 \%$ & $25 \%$ & $19 \%$ & $15 \%$ \\
\hline atención parece fluctuar una & 15 & 51 & 17 & 9 & 8 \\
\hline $\begin{array}{c}\text { y otra vez entre lo que } \\
\text { tengo que hacer y otras } \\
\text { cosas }\end{array}$ & $15 \%$ & $51 \%$ & $17 \%$ & $9 \%$ & $8 \%$ \\
\hline $\begin{array}{l}\text { 41.A menudo pierdo la } \\
\text { concentración durante la } \\
\text { competición por } \\
\text { preocuparme o ponerme a } \\
\text { pensar en el resultado final }\end{array}$ & $\begin{array}{c}16 \\
16 \%\end{array}$ & $\begin{array}{c}40 \\
40 \%\end{array}$ & $\begin{array}{c}33 \\
33 \%\end{array}$ & $\begin{array}{c}10 \\
10 \%\end{array}$ & $\begin{array}{c}1 \\
1 \%\end{array}$ \\
\hline $\begin{array}{l}\text { facilidad en aquello que es } \\
\text { lo más importante en cada } \\
\text { momento de una } \\
\text { competición }\end{array}$ & $\begin{array}{c}0 \\
0 \%\end{array}$ & $\begin{array}{c}0 \\
0 \%\end{array}$ & $\begin{array}{c}5 \\
5 \%\end{array}$ & $\begin{array}{c}59 \\
59 \%\end{array}$ & $\begin{array}{c}36 \\
36 \%\end{array}$ \\
\hline
\end{tabular}

Tabla 4

Medias y Desviación Típica de las variables psicológicas

\begin{tabular}{lcc}
\hline & Media & Desviación Estándar \\
\hline Ansiedad & 2.05 & .716 \\
Atención-Concentración & 2.21 & .498 \\
Autoconfianza & 3.47 & .502 \\
\hline
\end{tabular}

psicológicos en el futbolista de rendimiento.

En una investigación con 49 futbolistas bolivianos pertenecientes a tres equipos profesionales, Pacheco \& Gómez (2005), tras la aplicación del CPRD, especifican qué variables psicológicas influyen más en el rendimiento deportivo de los jugadores en función de sus demarcaciones particulares - porteros, defensas, centrocampistas y delanteros Los autores destacan el control del estrés como segunda variable con mayor incidencia en los porteros y defensas, y como predominante en los delanteros. En nuestro estudio, los porteros y delanteros son los que destacan en puntuaciones más altas en la variable control del estrés.

El trabajo de Fenoy \& Campoy (2012) sobre la relación entre la evitación experiencial en el deporte y las variables psicológicas vinculadas al rendimiento deportivo, fue llevada a cabo en 64 futbolistas con una edad media de 16 años y pertenecientes al Club Deportivo Vera (Almería). Para el análisis de las variables psicológicas se utilizó el CPRD, y tras analizar cada una de las escalas del instrumento se llegó a la conclusión de que el control del estrés ofreció puntuaciones que determinaban factores de la conducta disfuncional, aconsejándose tratamiento especializado para combatir este tipo de conductas. En nuestro estudio, el análisis del control del estrés en los futbolistas evaluados, muestra que ante situaciones deportivas erradas descienden sus niveles de atención-concentración, lo cual indica la importancia del trabajo específico de estas situaciones en prevención de ejecuciones erráticas posteriores. Sería preciso poner en práctica programas de entrenamiento adaptados al fútbol en el que se estructuren ejercicios de desarrollo de estilos atencionales específicos de manera inherente al entrenamiento integral del deportista (Mora, Zarco \& Blanca, 2001).

La utilización del CPRD en el ámbito deportivo es internacionalmente aplicada hasta nuestros días y múltiples estudios confirman su eficacia aportando datos significativos a la comunidad científica, así es el caso de Gimeno, Buceta \& Pérez-Llantada (2007) que descubren en 108 futbolistas de seis equipos de infantil, cadete y juvenil que a mayor puntuación en control del estrés, menor tasa de abandono, por lo que el análisis de esta variable puede predecir la continuidad del futbolista en el futuro. Nuestra investigación ha utilizado este instrumento para la obtención de información de los futbolistas estudiados en cuanto a la variable control del estrés, y los resultados revelan que controlan el estrés precompetitivo y durante la competición, pudiéndose anticipar una mayor continuidad deportiva de estos futbolistas en el futuro.

Tras una revisión de estudios científicos sobre la incidencia de la autoconfianza y el control del estrés en futbolistas, González-Campos 
et al. (2015) llegan a la conclusión de que se puede predecir el riesgo de vulnerabilidad a la lesión si el jugador registra altos niveles de ansiedad, o lo que es lo mismo, puntuaciones bajas en control del estrés. Resultados similares se encontraron en el estudio de Valdivia-Moral et al. (2016) en judocas chilenos. Nuestro estudio revela altas puntuaciones en control del estrés, por lo que se presume que los jugadores evaluados pudieran presentar menor vulnerabilidad a lesionarse.

El mismo estudio de González-Campos et al. (2015) confirma que a mayor nivel de autoconfianza en el futbolista, menor nivel de ansiedad, observando en el jugador un mayor control del estrés. Nuestro estudio coincide plenamente con estas conclusiones, pues los resultados demuestran que los jugadores analizados señalan bajas puntuaciones en ansiedad, al mismo tiempo que poseen altos niveles de autoconfianza.

\section{Conclusiones}

En base a los objetivos planteados y los resultados expuestos, se establecen las siguientes conclusiones:

- La mayoría de los futbolistas evaluados no padecen estados de ansiedad precompetitiva, por lo que no requieren tratamiento especializado en esta variable.

- Los niveles de autoconfianza son altos en los sujetos estudiados, si bien es recomendable seguir trabajando esta habilidad para mantener el nivel óptimo que presentan.

- Los deportistas analizados muestran altos niveles de atención - concentración durante la competición, sin embargo cuando cometen un error, manifiestan un descenso en esta habilidad psicológica.

- El estudio realizado indica que los futbolistas semiprofesionales analizados controlan el estrés precompetitivo y durante la competición, de manera que, en su mayoría no alcanzan estados de ansiedad, desarrollando altos niveles de autoconfianza y de atención-concentración.

Así pues como conclusión final, el estudio revela según la muestra seleccionada, que tener desarrollada la habilidad psicológica de controlar el estrés, previene alcanzar estados de ansiedad y como consecuencia, posibilita mantener elevada la autoconfianza y una buena capacidad de atención-concentración durante la competición.

\section{Referencias}

Buceta, J.M., Gimeno, F. y Pérez-Llantada, M.C. (1994). Cuestionario de Características Psicológicas relacionadas con el Rendimiento Deportivo (CPRD). Universidad Nacional de Educación a Distancia. (Inédito).

Buceta, J., López, A., Pérez-Llantada, M., Vallejo, M. y Del Pino, M. (2003) Estado psicológico de los corredores populares de maratón en los días anteriores a la prueba. Psicothema, 15(2), 273-277.

Cachón-Zagalaz, J., Rodrigo-Conde, M., Campoy-Aranda, T., Linares-Girela, D. y Zagalaz-Sánchez, M.L. (2012). Fútbol Sala y educación. Aprendizaje de un deporte colectivo para los escolares. Journal of Sport and Health Research, 4(3), 145-254.

Chacón, R., Castro-Sánchez, M., Espejo-Garcés, T. y Zurita, F. (2016). Estudio de la resiliencia en función de la modalidad deportiva: fútbol, balonmano y esquí. Retos. Nuevas Tendencias en Educación Física, Deportes y Recreación, 29 , 157-161.

Cox, R. H. (2009). Psicología del Deporte. Conceptos y sus aplicaciones. $6^{a}$ Edición. Madrid: Editorial Médica Panamericana

De la Vega, R., Rivera, O. y Ruiz, R. (2011). Personalidad resistente en carreras de fondo: Comparativa entre ultra fondo y diez kilómetros. Revista de Psicología del Deporte, 20(2), 445-454.

Dosil, J. (2004). Psicología de la actividad física y del deporte. Madrid: McGrawHill.

Dramisino, H. (2007). Resiliencia y deporte. Sinopsis Apsa Revista, 23(43), 23-25 Ferreira, M.R., Valdés, H.M. y González, E. (2002). Estrés en jugadores de fútbol: Una comparación Brasil y Cuba. Cuadernos de Psicología del Deporte, 2(1), $7-14$

García, S., Rodríguez, A. y Garzón A. (2011). Conceptualización de inteligencia táctica en fútbol: Consideraciones para el desarrollo de un instrumento de evaluación en campo desde las funciones ejecutivas. Cuadernos de Psicología del Deporte, 11, 69-78.

García-Naveira, A. y Ruiz-Barquín, R. (2015). Adaptación del I.S.R.A-B a la población deportiva a través de jugadores de fútbol de rendimiento. Ansiedady Estrés, 21(1), 83-94.
Gimeno, F., Buceta, J.M. y Pérez-Llantada, M.C. (2007). Influencia de las variables psicológicas en el deportede competición: Evaluación mediante el cuestionario características psicológicas relacionadas conel rendimientodeportivo. Psicothema, 19(4), 667-672.

González, G (2011). Aspectos tácticos y psicopedagógicos a tener en cuenta en el desarrollo deportivo de un partido de fútbol de relevancia institucional. Journal of Sport and Health Research, 3(1), 91-96.

González, J., Garcés de los Fayos, E. y Ortega, E. (2014). Avanzando en el camino de diferenciación psicológica del deportista. Ejemplos de diferencias en sexo y modalidad deportiva. Anuario de Psicología, 44(1), 31-44.

González-Ramírez,M., Quezada-Berumen,L.,Díaz-Rodríguez, C. y Cano-Vindel, A. (2014). Adaptación para México y estructura factorial del Inventario de Situaciones y Respuestas de Ansiedad Breve (ISRA-B). Ansiedad y Estrés, 20(1), 89-100.

González-Campos, G., Valdivia-Moral, P., Zagalaz, M.L. y Romero, S. (2014). Estudio sobrelas habilidades psicológicas en el futbolista. Trances: Revista de Transmisión del Conocimiento Educativo y de la Salud, 6(4), 227-248.

González-Campos, G., Valdivia-Moral, P., Zagalaz, M.L. y Romero, S. (2015). La autoconfianza y el control del estrés en futbolistas: revisión de estudios. Revista Iberoamericana de Psicología del Ejercicio y el Deporte, 10(1), 95-101.

Gucciardi, D.F., Gordon, S. y Dimmack, J.A. (2009). Development and preliminary validation of a mental toughness inventory for Australian football. Psychology of Sport and Exercise, 10(1), 201-209.

Hosseini, S.A., y Besharat, M.A.(2010). Relation of resilience whitsport achievement and mental health in a sample of athletes. Procedia Social and Behavioral Sciences, 5, 633-638.

Kent, M. (2003).Diccionario Oxford de medicina y ciencias del deporte. Barcelona: Paidotribo.

López-Suárez, M. R. (2014). Relación entre satisfacción con la vida y satisfacción con el deporte y en jóvenes deportistas. Tesis Doctoral: Universidad de las Palmas de Gran Canaria.

Lundqvist, C., Kenttä, G. y Raglin, J. S. (2011). Directional anxiety responses in elite and subelite young athletes: intensity of anxiety symptoms matters. Scandinavian Journal of Medicine and Science in Sports, 21(6), 853-862.

Moore, R., Bullough, S., Goldsmith, S. y Edmondson, L. (2014). Psychological elements of training and competition. American Journal of Sports Science and Medicine, 2(3), 108-116.

Mora, J.A., Zarco, J.A. y Blanca, M. J. (2001). Atención-Concentración como entrenamiento para la mejora del rendimiento deportivo en jugadores profesionales de fútbol. Revista de Psicología del Deporte, 10(1), 49-65.

Navlet, M.R. (2012). Ansiedad, estrés y estrategias de afrontamiento en el ámbito deportivo: Un estudio centrado en la diferencia entre deportes. Tesis Doctoral. Universidad Complutense de Madrid.

Nicolás,A. (2009). Autoconfianza y deporte. Lecturas, Educación Físicay Deporte. RevistaDigital. Recuperadode http://www.efdeportes.com/efd128/autoconfianzay-deporte.htm.

Nunnally, J. C. (1978). Psychometric Theory. New York: McGraw-Hill.

Pacheco, M. y Gómez, J. (2005). Características psicológicas y rendimiento deportivo. Un estudio en jugadores bolivianos de fútbol profesional. Ajayu, 3(2), 126.

Pazo, C.I., Sáenz-López, P.y Fradua, L. (2012). Influencia del contexto deportivo en la formación de los futbolistas de la selección española de fútbol. Revista de Psicología del Deporte, 21(2), 291-299.

Romero, C. E. (2015). Meta-análisis del efecto de la actividad física en el desarrollo de la resiliencia. Retos, (28), 98-103.

Ruiz, R., De la Vega, R., Poveda, J., Rosado, A. y Serpa, S. (2012). Análisis psicométrico de la escala de resiliencia en el deporte del fútbol. Revista de Psicología del Deporte, 21(1), 143-151.

Ruiz-Juan, F. y Zarauz,A. (2013). Análisis de la ansiedad en el atletismo: un estudio con veteranos. RICYDE. Revista Internacional de Ciencias del Deporte,33(9), 222-235. http://dx.doi.org/10.5232/ricyde2013.03302

Ruiz-Juan, F. y Zarauz, A. (2014). Ansiedad en maratonianos en función de variables socio-demográficas. Retos. Nuevas Tendencias en Educación Física, Deportes y Recreación, 25, 28-31.

Saz,A.I. (2000). Diccionario de Psicología. Madrid: Libro Hobby-Club.

Telletxea, S. (2007).Aplicación de un programa de intervención psicosocial orientado hacia la formación yoptimización de las capacidades de rendimiento en deportistas. Tesis Doctoral. Universidad del País Vasco.

Valdivia-Moral, P., Zafra, E., Zurita, F., Castro-Sánchez, M., Muros, J.J. y CofreBolados, C. (2016). Niveles de Ansiedad en Judocas chilenos. Journal of Sport and Health Research, 8(2), 129-138.

Vigário, I., Serpa, S. y Rosado, A. (2009). Tradução e adaptação da Escala de Resiliência para a população portuguesa. Universidade Técnica de Lisboa. Facultade de Motricidad Humana. Pendiente de publicación.

Weinberg, R. S. y Gould, D. (2010). Fundamentos de Psicología del deporte y el ejercicio físico. $4^{a}$ Edición. Madrid: Editorial Médica Panamericana. 